\title{
Le lexique mathématique breton : bilan et perspectives
}

De l'obsolescence à la revitalisation

The Breton mathematical lexicon: a review and prospects

\section{Erwan Le Pipec}

\section{(2) OpenEdition}

\section{Journals}

Édition électronique

URL : http://journals.openedition.org//bl/602

DOI : $10.4000 /$ lbl.602

ISSN : 2727-9383

\section{Éditeur}

Université de Bretagne Occidentale - UBO

Édition imprimée

Date de publication : 1 octobre 2018

Pagination : 41-75

ISBN : 979-10-92331-40-0

ISSN : 1270-2412

\section{Référence électronique}

Erwan Le Pipec, "Le lexique mathématique breton : bilan et perspectives », La Bretagne Linguistique [En ligne], 22 | 2018, mis en ligne le 01 mai 2020, consulté le 01 octobre 2020. URL : http:// journals.openedition.org//bl/602 ; DOI : https://doi.org/10.4000//bl.602

La Bretagne Linguistique est mise à disposition selon les termes de la Licence Creative Commons Attribution 4.0 International. 


\section{Le lexique mathématique breton : bilan et perspectives}

\section{De l'obsolescence à la revitalisation}

C

omme beaucoup de langues minoritaires, ce n'est qu'à une date récente que le breton a accédé au statut de langue de scolarisation ${ }^{1}$. Un tel état de fait conduit souvent à mettre en avant la responsabilité de l'État, organisateur méthodique de l'exclusion du breton, au nom d'une glottopolitique assimilationniste. Sans revenir en détail sur cette question, force est cependant de constater que l'introduction du breton à l'école a longtemps été rendue impossible par un blocage psychologique et culturel chez les Bretons eux-mêmes. Blocage qui les conduisait à percevoir le breton, en dépit de l'attachement qu'ils pouvaient lui porter, comme une langue inapte à remplir un certain nombre de fonctions, en particulier l'accès au savoir et à l'écrit ${ }^{2}$. L'exclusion de l'école en découlait naturellement, privant le breton du statut de langue de culture

* Maitre de conférences de breton, UBO-CRBC (EA 4451 / UMS 3554).

1. Fañch Broudic, Parler breton au XXIe siècle, Brest, Emgleo Breiz, 2009 ; Gilles PrÉMEL, "Anamnèse d'un dommage, ou comment le français et venu aux Bretons », Langage et société, t. 72, 1995.

2. Daniel BERNARD, «La langue bretonne à l'école primaire : un projet officiel d'enseignement bilingue en Basse-Bretagne en $1831 »$, Annales de Bretagne, t. 32-1, 1917 ; Fañch ROUDAUT, «La littérature religieuse en breton », dans J. Balcou, \& Y. Le Gallo (dir.), Histoire littéraire et culturelle de la Bretagne, t. I, Paris, Champion et Spézet, Coop Breizh, 1997. 
et venant ainsi entériner et renforcer le postulat de départ. Solide dans une société figée et indifférente à la scolarisation de ses enfants, le breton s'est soudainement effondré quand il a été perçu comme une entrave par des populations rurales en forte mobilité sociale, devenues conscientes de l'enjeu de la scolarisation et qui adoptaient les normes de comportement urbaines ${ }^{3}$.

L'exclusion de l'école n'a cependant pas eu que des conséquences externes : le corpus même de la langue en a été affecté. Les locuteurs ayant été équipés d'outils intellectuels et d'une culture scolaire par le canal exclusif du français, le breton a fini par s'appauvrir. Soit parce que les termes techniques dont il disposait ont peu à peu été oubliés (au profit d'emprunts ou de code-switching vers le français); soit parce que les termes nouveaux, la phraséologie, indispensables pour adapter la langue à un usage scolaire et qu'il aurait convenu de forger ne l'ont pas été. Insensiblement, le breton a donc glissé vers ce que Swadesh ${ }^{4}$ appelle l'obsolescence, état combinant divers degrés de délabrement interne et d'inadaptation sociétale.

De cette manière, le breton prend place parmi les très nombreuses langues menacées de la planète. Il s'agit là d'un champ d'investigation dont les bases ont été posées il y a plusieurs décennies par différents courants de la sociolinguistique (de Fishman à l'école catalano-occitane) et par l'anthropologie linguistique ${ }^{5}$. Mais le domaine reste encore en chantier : renouvelé au début des années $1990^{6}$, sa terminologie n'est pas bien stabilisée (langues minorées, langues menacées, langues en danger, threatened languages, endangered languages...). Où se rencontrent de nombreuses définitions et typologies, tant des causes que des réponses à y apporter : revitalisation, revernacularisation, normalisation, language revival, reversing language shift, etc. ${ }^{7}$.

Parmi ces termes et concepts, celui de revitalisation semble s'imposer. Lequel ne doit pas être compris nécessairement comme une tenta-

3. Erwan LE PIPEC, «Les trois ruptures sociolinguistiques du breton», International Journal of the Sociology of Language, t. 223, 2013.

4. James COSTA, Revitalisation linguistique : discours, mythe et idéologie (thèse), Université Stendhal-Grenoble III, Grenoble, 2010, p. 32.

5. Ibidem.

6. Ibid. p. 36.

7. Ibid. p. 54-56. 
tive de restauration d'un usage sociétal dominant (ce qui pour certaines langues peut être devenu hors de portée), mais au moins dans une première étape, comme l'attribution d'un statut de «langue-trésor ${ }^{8}$, patrimoine légitime de tous, quand bien même elle ne serait parlée que par une fraction de la population. De telles conditions semblent réunies en Bretagne, où il existe une demande sociale quant à la préservation de la langue ${ }^{9}$. Néanmoins, il est clair que pour y parvenir, la revalorisation statutaire (status planning) n'est pas suffisante. Celle-ci n'est qu'une focale, au sein d'une stratégie d'ensemble, qui inclut également un travail d'ajustement interne (corpus planning); une politique de transmission, notamment par l'école si la transmission familiale est trop limitée (acquisition planning); et un usage volontariste visant à rendre la langue-cible présente dans tous les domaines (usage planning ou normalisation selon la terminologie catalane).

\section{À l'origine d'une recherche...}

Le présent article livre donc une réflexion qui s'inscrit dans une démarche de corpus planning ${ }^{10}:$ d'évaluation des ressources internes de la langue, dans un but d'élaboration et d'affinement des outils afin de les rendre les plus pertinents possible dans le cadre d'un enseignement en breton. Il fait suite aux travaux menés dans le cadre d'un groupe de recherche en éducation à l'ESPE de Bretagne : MELBA (mathématiques à l'école en langue bretonne aujourd'hui), qui a fédéré une assez large équipe de chercheurs et enseignants de terrain entre 2013 et 2015 et qui nous a permis d'aller à la rencontre des pratiques enseignantes ${ }^{11}$.

8. Colette GRINEWALD \& Bert MiCHEL, «Langues en danger, idéologies, revitalisation », Cabiers de l'Observatoire des pratiques linguistiques 3 (DGLFLF), 2012, p. 29.

9. Fañch Broudic, op. cit., p. 143 ; Erwan LE PIPEC, « Le breton, langue-totem ? ", La Bretagne linguistique, vol. 18, 2013.

10. Colin BAKER, Foundations of Bilingual Education and Bilingualism, Clevedon, Multilingual Matters, 2011, p. 52.

11. Martine Kervran, Máire Ní Ríordáin \& Erwan Le PiPEC, "Significant insights into Teaching and Learning Mathematics in Bilingual Classrooms: Examples from Brittany, Catalonia, New Zealand and Ireland », Table ronde à l'ECER (European Conference on Educational Research), Istanbul, 10-13 septembre 2013 ; Martine Kervran, Caroline Poisard, Erwan Le PiPeC, Marianne Sichler, \& Nathalie JEUdyKARAKOÇ, «Langues minoritaires locales et conceptualisation à l'école : l'exemple de l'enseignement des mathématiques en breton », dans M. Kervran \& Ph. Blanchet (dir.), 
C'est dans ce contexte qu'a pu être dressé le constat d'une certaine imprécision de la langue, par la fréquente concurrence entre différents termes, qui ne permet pas l'expression univoque. Ceci dans un domaine, les mathématiques scolaires, où elle apparaît pourtant fondamentale. Comment dire géométrie en breton, si tel manuel propose mentonies $b^{12}$ et tel autre geometriez ${ }^{13}{ }^{13}$ ? Plus surprenant encore, la terminologie mathématique est même parfois contradictoire : en 1998, la première édition du Geriaoueg matematik ${ }^{14}$ traduit kensturieg par parallélogramme. En 2005, dans la réédition du même ouvrage par le même éditeur, kensturieg signifie parallélépipède ${ }^{15}$. Cette inconstance du vocabulaire peut s'expliquer par la rotation des traducteurs, par la concurrence de vues didactiques opposées mais aussi par de réelles avancées dans la réflexion sur la traduction (notamment grâce à la publication égrenée dans le temps, selon les niveaux, de plusieurs manuels ou encore la publication du dictionnaire des mathématiques de J. Marot en $2002^{16}$ ). Mais assez naturellement, ces flottements sont ressentis comme une

Langues minoritaires locales et éducation à la diversité, des dispositifs didactiques à l'épreuve, Paris, L'Harmattan, 2015 ; Caroline PoISARD, Martine Kervran, Erwan LE PIPEC, Stefan Alliot, Ghislaine Gueudet, Hélène Hili, Nathalie Jeudy-Karakoç \& Gwénolé LARVOL, «Enseignement et apprentissage des mathématiques à lécole primaire dans un contexte bilingue breton-francçais ", Spirale, t. 54, 2014 ; Caroline POISARD, Máire Ní RíORDÁIN \& Erwan LE PIPEC, "Mathematics education in bilingual contexts: Irish-English, Breton-French», dans K. Krainer \& N. Vondrová (dir.), Proceedings of the 9th Congress of the European Society for Research in Mathematics Education, Prague, Université Charles, Faculté des sciences de l'éducation \& ERME, 2015 ; Erwan LE PIPEC, « Mathematical vocabulary in Breton: assessment and prospects », 9th Celtic Linguistics Conference, Cardiff University, $1^{\mathrm{er}}-2$ septembre 2016.

12. Penaos 'mañ kont? Klas 5 (Saint-Brieuc, TES, 1995), traduction/adaptation du manuel Le nouvel objectif calcul CM2 (Paris, Hatier, 1988 et 1995).

13. War hent ar matematik CM2 (Saint-Brieuc, TES, 2013), traduction/adaptation du manuel Cap Maths CM2 (Paris, Hatier, 2010).

14. Lexique mathématique. Il s'agit d'un supplément reprenant le lexique utilisé dans la collection des manuels Penaos 'mañ kont? éditée par TES (cf. notes ci-dessus).

15. Bien que reprenant le même titre et la même maquette, ce lexique présente cette fois les termes utilisés dans plusieurs publications de TES : la collection Mat da zeskin, traduction/adaptation de J'apprends les maths (Paris, Retz, 2000) ; les manuels de $6^{\mathrm{e}}-5^{\mathrm{e}}$; et l'outil d'algèbre Lieskementout, traduction/adaptation de Multiplicatou (Paris, Nathan, 2002).

16. Jean MAROT, Geriadur ar matematikoù - dictionnaire multilingue des mathématiques, Morlaix, Skol Vreizh, 2002. 
gêne par de nombreux enseignants. Souvent en relative insécurité professionnelle, soit du fait de leur statut (vacataires remplaçants de longue durée), soit du fait d'une représentation hésitante du modèle didactique qu'il leur faut inventer en classe bilingue, après qu'ils aient eux-mêmes été le plus souvent scolarisés en classe monolingue, ils se passeraient bien d'une insécurité linguistique. Surtout dans cette matière particulièrement anxiogène pour les parents d'élèves. S'ils acceptent l'argument d'autorité du manuel, encore demandent-ils à comprendre pourquoi les mots qu'ils employaient il y a quelques années ne sont plus acceptables aujourd'hui ${ }^{17}$. D'autant que les enseignants sont exposés à une multitude de sources non concordantes : outre les manuels édités par TES ${ }^{18}$, on trouve encore dans certaines écoles de vieux polycopiés des années 1990, où les termes employés peuvent être différents. Le développement de l'informatique et d'internet ajoute encore à la prolifération : de nombreux enseignants proposent ainsi à leurs collègues des ressources en ligne qu'ils ont eux-mêmes mises au point, où les choix lexicaux reflètent le plus souvent un certain empirisme, voire de l'improvisation, plutôt qu'une stratégie réfléchie. Comment interpréter autrement jeometrię̧, autre traduction usuelle pour géométrie ${ }^{19}$ ? Et même si stratégie il y a, elle est dépendante des compétences linguistiques et scientifiques des enseignants, elles-mêmes très variables selon leur parcours d'études ${ }^{20}$. On comprend donc la perplexité certaine de nombreux enseignants : que transmettre aux élèves? Y a-t-il un intérêt didactique à privilégier un vocable plutôt qu'un autre, etc. ?

17. Entretiens dans le cadre de MELBA non publiés.

18. TES, pour Ti Embann ar Skolioù (Editions des écoles), département de Canopé (exCRDP de Bretagne) spécialisé dans les ouvrages en breton et qui approvisionne les écoles en manuels scolaires, souvent traduits du français. Sa politique éditoriale est déterminée par un conseil d'édition où siègent notamment des inspecteurs de l'éducation nationale.

19. Vu sur le site webklas.org.

20. Mentionnons enfin qu'il existe parfois de fortes têtes qui ne sont pas d'accord avec les équivalents de traductions les plus répandus et qui bricolent dans leur coin leurs propres terminologies... avec plus ou moins de bonheur! Ce cas de figure semble pourtant devenu rare. 


\section{Protocole d'observation}

En s'en tenant à cet aspect lexical, la première tâche a donc paru être de mesurer l'étendue de la variation dans le registre mathématique du breton. Ensuite, de comprendre pourquoi une telle variation existe. Enfin, pour ne pas perdre de vue le lien avec le terrain et les demandes formulées par les enseignants, de proposer éventuellement des recommandations, selon que la variation aura été jugée pertinente ou non en elle-même, ou selon que tel ou tel terme aura paru mériter la préférence.

Après différentes tentatives exploratoires, c'est un échantillon de vingt-quatre termes mathématiques qui a donc été isolé, en raison de leur usage courant à l'école dans différents domaines des mathématiques : numération et calcul, géométrie et grandeurs et mesures :

\begin{tabular}{|c|c|}
\hline Numération et calcul & $\begin{array}{l}\text { nombre, numération, calcul, opération, } \\
\text { addition, soustraction, multiplication, } \\
\text { retenue, somme, unités, dizaines, centaines, } \\
\text { chiffres, fraction }\end{array}$ \\
\hline Géométrie & $\begin{array}{l}\text { carré, cercle, rectangle }{ }^{21} \text {, octogone, } \\
\text { losange, parallélogramme, parallélépipède }\end{array}$ \\
\hline Grandeurs et mesures & aire, périmètre, diamètre \\
\hline
\end{tabular}

Pour dresser la liste de leurs équivalents en breton, j’ai eu recours aux deux lexiques mentionnés précédemment, à savoir les deux versions du Geriaoueg matematik, édités par TES en 1998 et 2005, et qui de ce fait représentent a priori la norme scolaire.

Afin de mieux comprendre leur périmètre d'usage, ceux-ci ont été comparés aux données de quatre dictionnaires en ligne, disponibles à partir du site Lexilogos et qui, par expérience, semblent abondamment consultés par les étudiants et les enseignants : celui de Francis Favereau $^{22}$ (désormais F) ; celui des éditions $\operatorname{Preder}^{23}$ (désormais P) ; la

21. Rectangle a été considéré deux fois : en tant que nom et qu'adjectif.

22. Professeur émérite de celtique à l'Université Rennes 2. Le dictionnaire en ligne est basé sur la version imprimée, publiée en 1992, mais ce n'est pas F. Favereau lui-même qui est à l'origine de sa mise en ligne.

23. Ce dictionnaire reprend également les bases de données ayant servi à de nombreux lexiques spécialisés édités sur papier depuis les années 1960. 
banque de données Termofis, administrée par l'Office public de la langue bretonne (désormais $\mathrm{O}$ ) ; enfin le dictionnaire des sciences et des techniques de l'association Kreizenn ar geriaouiñ ${ }^{24}$ (désormais $\mathrm{K}$ ).

Enfin, pour donner plus de profondeur à l'analyse, pour mieux comprendre la généalogie des termes et des choix qui ont présidé à leur élaboration, ils ont également été comparés à ceux fournis par trentehuit dictionnaires et ouvrages divers, publiés entre 1450 et 2012 (cf. annexe).

\section{Premiers constats}

Comme le montre le tableau 1 en annexe, sur les vingt-quatre termes répertoriés, seuls cinq font l'unanimité : nombre (niver), soustraction (lamadenn/ lamadur), unité (unanenn), chiffre (sifr), carré (karrezenn).

Le consensus est toutefois presque atteint pour dix autres termes. Soit qu'il y ait une entrée supplémentaire dans une seule source : sammadenn/sammadur ${ }^{25}$ traduisent addition dans les six dictionnaires et lexiques, mais chez TES avec un doublet sommadenn; de même, multiplication est traduit par liesadenn/liesadur, avec ajout de lieskementadenn/lieskementadur chez TES en 1998 ; pour cercle, c'est kelc'b qui apparait dans toutes les sources, mais les quatre dictionnaires en ligne, et eux seuls, lui adjoignent kerlenn. Soit à l'inverse, une seule source est silencieuse : centaine est traduite par kantad par tous sauf $\mathrm{O}$, qui reste muet ; pour aire, tous donnent gorread, sauf F, qui ne donne pas de sens mathématique. La quasi-unanimité concerne également des mots qui ne diffèrent que par une variation de suffixe sans impact sur le sens : numération (niveradur/ nivererezh) ; retenue (dalc'h/dalc'had) ; calcul (jedad/jedadenn) et dizaine (deg/ degad) ; ou encore par la présence de doublets quasi-homophones : somm et soumm (pour somme) chez F.

Restent donc dix termes présentant d'importantes divergences. Pour quatre d'entre eux, on observe des essais de néologie à partir du breton, auxquels $\mathrm{F}$ ajoute des emprunts directs au français (avec bre-

24. Centre de terminologie. L'association, regroupant essentiellement des enseignants et universitaires, a été créée en 1985 pour fournir aux enseignants du réseau Diwan les terminologies qui leur manquaient.

25. Le breton distingue entre l'opération effectuée, exprimée par le suffixe -adenn et le processus d'additionner, de soustraire de multiplier, grâce au suffixe -adur. 
tonnisation de la graphie) : opération se traduit ainsi par oberiadenn (TES), jedadenn $(\mathrm{K}$ et $\mathrm{F})$, gwezhiadenn $(\mathrm{P})$ ou operasion $(\mathrm{F})$. Pour fraction, on relève kevrenn (TES et $\mathrm{K}$ ), rann (P et $\mathrm{F}$ ) ou fraksion $(\mathrm{F})$. Le nom rectangle est traduit par skouergorneg ${ }^{26}(\mathrm{TES})$, skouergorn $(\mathrm{O})$, reizhkogn $(\mathrm{K})$, reizhkorn $(\mathrm{P})$, reiz̧bkorneg $(\mathrm{P})$, hirgarrez $(\mathrm{F})$ et rektankl $(\mathrm{F})$. L'adjectif donne à peu près les mêmes entrées ${ }^{27}$, auxquelles il faut ajouter $\operatorname{skwer}(\mathrm{K})$ et $\operatorname{ser} \not b(\mathrm{P})$. Enfin, six termes manifestent de véritables stratégies lexicologiques nettement opposées, entre recours à la néologie à base celtique pour les uns, gréco-latine pour les autres : octogone est eizhkorneg ou eizhkostezeg chez TES ; eizhkorn et eizhtueg chez P ; eizhkoogn chez F, mais oktogon chez K. Losange se traduit par lankell chez $\mathrm{P}$, face à romb chez TES, O et K, et lozañj chez K et F. Pour parallélogramme, TES donnait kensturieg en 1998, comme P. Mais en 2005, c'est parallelogram, tout comme K et F. Pour parallélépipède, TES donne kensturdaleg en 1998, puis y ajoute kensturieg en 2005 ; O et P donnent kensturdaleg; en 2005, TES donne également parallelepiped, auquel se rangent $\mathrm{K}$ et $\mathrm{F}$ (mais avec un seul «1» chez F, qui cite également " pezh c'hwec'h tu ${ }^{28}$ ). Périmètre remporte la palme de la diversité, avec trolinenn et trohed chez TES ; amregad et amregenn chez $\mathrm{P}$; muzul-tro chez $\mathrm{F}$ et perimetr chez $\mathrm{K}$. Mais diamètre montre aussi beaucoup de variations, avec treur (F), treuzkiz. (O, P et TES en 1998), treuzkizenn (TES en 1998), opposés à diametr chez TES en 2005, ainsi que chez $\mathrm{K}$ et $\mathrm{F}$.

\section{Pourquoi une telle variation?}

La terminologie mathématique en breton est donc, à tout le moins, mal unifiée. Or, lorsque l'on se penche sur les termes qui présentent le degré le plus élevé de variation, deux caractéristiques émergent, qui pourraient en expliquer la raison. On peut ainsi observer tout d'abord que c'est le vaste domaine de la géométrie et des grandeurs et mesures qui paraît le plus disparate. Seuls les noms du cercle et du carré sont largement partagés, c'est-à-dire les formes les plus élémentaires. De

26. La graphie est parfois instable : skouergorneg et skwergorneg chez TES en 2005.

27. Au détail près de l'alternance régulière en graphie peurunvan entre terminaisons - $-g$ pour les noms et -ek pour les adjectifs.

28. Pièce à six côtés. Cette périphrase semble le développement d'une proposition apparue en 1986, cf. infra. 
même, il y a aussi une certaine élémentarité chez les autres termes qui forment un consensus parfait ou relatif : chiffre, unité, nombre, addition, retenue, dizaine... Est-ce à dire que l'importance de la variation serait liée au degré d'élaboration et d'abstraction d'un concept ? Faut-il recourir à l'hypothèse Sapir-Whorf et conclure que le breton est une langue particulièrement apte à exprimer des nuances liées à la perception abstraite de l'espace ? La réalité semble plus prosaïque. D'autant que la prolifération de variantes ne résulte pas forcément du fonds indigène de la langue, mais de nombreuses adaptations à partir du grec ou du français. En fait, et c'est là la deuxième caractéristique frappante du corpus, les termes largement partagés ont surtout en commun d'être en usage en breton depuis une époque assez, voire très ancienne : l'Antiquité s'agissant de niver (nombre), issu du latin numerus, ou de kelc'b (cercle), venant du vieux-breton circhl, lui-même du latin circulus. En revanche, les termes les moins consensuels apparaissent tendanciellement de création récente, voire très récente: lankell (losange) n'est attesté que depuis 2005. Ce n'est donc pas dans les propriétés internes de la langue, mais dans son histoire externe qu'il faut rechercher la source de l’instabilité.

La plupart des termes mathématiques bretons furent en effet créés ou reçurent leur acception spécifiquement mathématique aux XIX et $\mathrm{XX}^{\mathrm{e}}$ siècles seulement. Ce fut le cas non seulement des termes de géométrie, mais également du domaine algébrique. Même le consensus qui semble atteint aujourd'hui autour de sammadenn, lamadenn et liesadenn pour exprimer les opérations basiques, addition, soustraction et multiplication, est fort récent. Bien que ces mots semblent limpides et pétris de bon sens, à partir des verbes : sammañ (additionner), lemel $^{29}$ (ôter) et de l'adverbe lies (souvent), trois mots dont l'usage est attesté depuis le XVe $\mathrm{s}$. Auparavant, pour addition/additionner, le premier dictionnaire breton, le Catholicon, donne bien en 1464 le verbe samaff (graphie équivalente au sammañ moderne), avec le sens de sommer, en latin summare, donc effectuer une somme. Mais en 1732, cette acception est rendue par le substantif cresquançz (augmentation) et la locution laquidiguez a névez, (ajout de surcroît), tandis que le verbe additionner est remplacé par une défi-

29. Infinitif irrégulier, qui se fléchit avec la base verbale lam-. 
nition : eus a veur a soum ober unan bepmuyqen (de plusieurs sommes n'en faire qu'une seule). Toujours dans le même ouvrage, samma n'est donné qu'avec son sens de la vie courante : charger un cheval, que les dictionnaires de 1821 à 1847 reconduiront. À partir de 1842 apparaît cependant ce qui ressemble à des tentatives pour gagner en précision, tout en restant concis. Addition est donc rendu par kresk (augmentation), jed (jeton), jedenn (idem) et niveridigez (alors qu'en 1821, ce dernier avait le sens de dénombrement). En 1847, c'est strobad (groupement), stroll et strollad (idem). En 1886, strollad est confirmé, mais additionner est traduit par renka* ${ }^{*}$ chiffr (ranger *des chiffres ${ }^{30}$ ). En 1919, c'est strolladur (action de grouper) ; en 1931, daspugnadur (idem); en 1934, kenstroll (idem); en 1937, kengrounnadur et kendaspugn (idem); en 1986, kont (compte) pour finir en 2002 avec adission. Le couple sammadenn/sammadur évoqué plus haut n'est adopté qu'en 1989, après une éclipse de son étymon de près de deux siècles. Mêmes flottements pour la soustraction : alors qu'en français, soustraction relève du registre mathématique depuis le XV siècle ${ }^{31}$, le mot est absent des dictionnaires bretons du XVII ${ }^{\mathrm{e}}$ siècle et n'apparaît au XVIII ${ }^{\mathrm{e}}$ qu'avec le sens moral lié au vol. En 1732 : enlevemen, recelemen de papiers, de meubles. Pour trouver trace de la soustraction mathématique, il faut donc se tourner vers l'entrée retranchement, enlevement d'une partie du tout, pour laquelle on lit : sqeigeadur, trouc'badur eus a lod, lamediguez: Tout au long du XIXe siècle, les dictionnaires reproduisent la même logique, avec les mêmes traductions, en ajoutant quelques variantes : krennadur (action de couper) et terridigez (brisure ?) en 1847 ; torridigez (idem) en 1886. Puis au XXe siècle, l'entrée soustraction prend son sens mathématique, mais les vieux termes énumérés auparavant ne sont plus mentionnés, remplacés par ce qui ressemble à autant de créations ad hoc. En 1919 : diskontadur (action de "décompter») ; en 1934 : diforc'b (différence); en 1937 : diforc'badur et diskontadur (idem avec suffixes d'action); ce dernier repris en 1986. Là aussi, ce n'est qu'en 1989 qu'apparaissent les actuels lamadenn/lamadur, qui font écho au lamediguez de 1732. Une litanie analogue pourrait être répétée pour la multiplication.

30. Avec omission de la désinence du pluriel dans l'exemple cité, qui aurait dû être renka chiffrou.

31. Centre national de ressources textuelles et lexicales (CNRS) : www.cnrtl.fr/etymologie/ soustraction. 
Il serait inutile de s'attarder sur une liste aussi exhaustive. Mentionnons simplement qu'en 1659, multiplier était traduit par crisqui (agmenter), aux côtés de multipliaff et angmenti. Ce verbe crisqui a pour corollaire les noms crisscance et crisscadur, attestés en 1744. Mais que le dictionnaire de 1732 (sous la forme cresquançz) traduisait par addition...

\section{Huit « écoles » lexicographiques}

Cette profusion de termes pourrait donner l'impression d'une certaine anarchie. Mais celle-ci n'est qu'apparente. Car lorsque l'on compare les différentes solutions proposées, qu'on les met en relation avec la personnalité de leurs concepteurs et l'époque à laquelle ils ont été actifs, des lignes de force transparaissent assez clairement. L'abondance des termes mathématiques semble ainsi le résultat tantôt de la succession, tantôt de la concurrence, parfois même de la guerre ouverte, entre différentes écoles et de leur projet pour la langue bretonne. Ce sont ainsi huit grands courants lexicologiques qui apparaissent :

1) Le breton à l'âge d'innocence (jusqu'au XVIII siècle) : les premiers lexicographes du breton n'avaient pas pour but de légiférer sur la langue, mais de faciliter aux clercs bretonnants soit l'apprentissage $\mathrm{du}$ latin et du français, soit le prêche dans la langue quotidienne du peuple $^{32}$. À partir du Catholicon de J. Lagadeuc (manuscrit de 1464, imprimé en 1499), ils vont ainsi avant tout représenter la langue telle qu'elle est réellement usitée : peu de termes techniques pointus (ou spécifiquement mathématiques), mais plutôt pour l'essentiel des mots de la vie courante, composant un vocabulaire étonnamment proche de la langue actuelle. L'origine des mots n'est pas une préoccupation, de sorte qu'au fonds celtique se greffent sans complexe les emprunts au français et au latin, surtout lorsque le besoin de termes abstraits se fait sentir (d'où multipliaff, angmenti, etc.). Les dictionnaires de 1626, 1659 et 1723 sont dans la même veine. Ceux de 1732 et 1744 également, mais ils se veulent plus encyclopédiques, d'où un plus grand nombre de

32. Christian GuYONVARC'H, «Du breton moyen au breton moderne », dans J. Balcou \& Y. Le Gallo, (dir.), op. cit., p. 202-203 ; Julien MAUNOIR, Le sacré collège de Jésus, Quimper, Jean Hardouyn, 1659, p. 7 ; Fañch ROUDAUT, «La littérature religieuse en breton », dans J. Balcou, \& Y. Le Gallo (dir.), op. cit., p. 234. 
termes abstraits et le recours à la description (souvent approximative) quand un mot fait défaut. Ainsi en 1744, un losange est un carré en déss er borne d'erblué (carré qui a le coin en haut).

2) Le projet romantique: restaurer la langue déchue (1807-1900) : à partir de la grammaire de Le Gonidec, en 1807, il n'est plus question de cantonner le breton au parler de paysans mal dégrossis. On croit se souvenir que le breton est aussi la langue des guerriers celtes du fond des âges ${ }^{33}$, qu'il faut s'efforcer de retrouver et de reconstituer pour lui rendre sa pureté originelle ${ }^{34}$. En 1821, le dictionnaire du même auteur inaugure donc de nouveaux principes : effort de rigueur, néologie exploitant les ressources de la langue, omission des emprunts français, emprunts (assez limités) au gallois. Niveridigez et nivererez. apparaissent ainsi pour dénombrement, le deuxième ayant aussi le sens d'énumération et de calcul. Multipliaff (multiplier) disparait, remplacé par muia et muiédigę (multiplication). Pour diamètre, l'édition françaisbreton de 1847, augmentée par La Villemarqué, précise : «En Galles, trévиұul». L'œuvre de Le Gonidec exercera une forte influence, mais ses successeurs seront parfois moins prudents que lui et continueront à créer des mots en se basant sur l'expérience courante. En 1886, du Rusquec ne semble pas distinguer un parallélogramme d'un losange (le premier est donné pour synonyme à l'article définissant le second), qu'il traduit par karré hir (carré long) ou même mouchouer (mouchoir)!

3) De Vallée à Gwalarn, l'élaboration d'une langue nationale (1900-1944): à la charnière des XIX ${ }^{e}$ et XXe siècles, les études sur la langue bretonne sont dominées par François Vallée. Par son volontarisme, il refonde la lexicologie bretonne, suscitant même une réforme de l'orthographe ${ }^{35}$. Rejoint en 1924 par les jeunes écrivains de Gwalarn $^{36}$, le mouvement

33. On décèle déjà plus ou moins ce type de motivation dans le dictionnaire de Le Pelletier (ms. de 1716, imp. en 1752), mais le projet de l'auteur restait descriptif.

34. Yves LE BerRe, La littérature de langue bretonne, livres et brocbures entre 1790 et 1918, Brest, Ar skol vrezoneg \& Emgleo Breiz, 1998, p. 501, 598 ; Bernard TanguY, « Des Celtomanes aux Bretonistes : les idées et les hommes », dans J. Balcou \& Y. Le Gallo (dir.), op. cit., p. 296, 316-321.

35. Iwan WMFFRE, Breton orthographies and dialects. The twentieth-century orthography war in Brittany, Oxford, Peter Lang, 2007.

36. Noroît, journal littéraire fondé par Roparz Hemon. 
prend une coloration de plus en plus nationaliste, voire mystique ${ }^{37}$. Grâce à la vaste érudition des auteurs (Vallée, Ernault, Hemon...), portés par un projet conquérant, on assiste en quelques années à une énorme production néologique. Le souci de rigueur est indéniable, mais il est parfois bousculé par une volonté encyclopédique, qui pousse à créer une foule de mots sans y consacrer la réflexion nécessaire : rectangle est traduit en 1931 dans le grand dictionnaire de Vallée par hirgarrezenn (long carré, entrée toujours présente en 2005, dans la 11édition du dictionnaire de Roparz Hemon). Les racines celtiques sont résolument privilégiées, par volonté de mobiliser les ressources de la langue, mais aussi dans certains cercles par rejet de la francité. Influence de l'espéranto (cher à Hemon) ou tropisme germanique, la suffixation et la préfixation sont abondamment utilisées. C'est le cas dans beskellgarręenn (carré oblique), pour losange, que Kerjean (alias Laîné) crée en 1934 et que Vallée adopte en 1937, abandonnant son karrezenn-war ${ }^{38}$ (carré arqué) de 1931. De cette époque datent également les exemples vus plus haut, liesadur (multiplication, 1937) et jedadenn (calcul, 1931).

4) La postérité de Vallée-Gwalarn, breton ou novlang? (1944-...) : après la compromission de certaines personnalités bretonnes durant l'Occupation, le discrédit retombe sur toute activité liée au breton. L'activisme lexicologique sera donc essentiellement le fait de militants isolés et dispersés, parfois exilés, donc de plus en plus coupés de la population bretonnante ${ }^{39}$. En 1958 et en 1962 sont créés les associations Preder ${ }^{40}$ et le SADED ${ }^{41}$. Tous deux prolongeront le courant précédent, en s'éloignant insensiblement de la langue effectivement parlée : leur vocabulaire de plus en plus riche et de plus en plus hermétique est encore à ce jour en construction permanente, mais très peu diffusé et parfois moqué (« breton chimique », « néo-breton » etc.). Le manuel grammatical

37. Yves Le Berre, Qu'est-ce que la littérature bretonne?, Rennes, Presses Universitaires de Rennes, 2006, p. 29-30 ; Fañch MorvanNOU, « La littérature de langue bretonne au $\mathrm{XX}^{\mathrm{e}}$ siècle, du début du siècle au second après-guerre : les hommes et les œuvres », dans J. Balcou \& Y. Le Gallo (dir.), op. cit., p. 208-211 ; Iwan WMFFre, op. cit., p. 33-35.

38. Karrezenn-war signifie toutefois parallélépipède chez Roparz Hemon en 1970.

39. Lukian KergoAT, «Terminologie bretonne : rétrospective, débats et enjeux », Recerc, t. 5, 2012.

40. Réflexion.

41. Strollad an Deskadurez̧h Eil Derez, Groupe de l'éducation du second degré. 
du moyen-breton de Goulven Pennaod (2e édition de 1977) offre ainsi l'illustration d'un breton très ésotérique. Martial Ménard, bon connaisseur de la langue populaire et auteur d'un dictionnaire paru en 2012, est plus mesuré. Il s'inscrit pourtant plus ou moins dans ce courant, par la filiation revendiquée avec Vallée et par la priorité qu'il donne aux néologismes d'origine celtique, avec parfois peu d'égards pour l'usage ${ }^{42}$.

5) Le lexique de Diwan, ambition, pragmatisme et éclectisme (1985-...) : le courant précédent représente en quelque sorte le versant dogmatique de l'héritage de Vallée-Gwalarn. Il existe toutefois également un courant plus pragmatique, plus favorable à l'adaptation en breton de termes empruntés aux diverses langues du monde via le français. C'était déjà une position défendue par Roparz Hemon, dont le dictionnaire est assez éclectique ${ }^{43}$. Ce sera également le parti pris de Per Denez (son dictionnaire de 1993 est toutefois un usuel pour débutants assez succinct). C'est ce pragmatisme, mêlé d'ambition pour le breton, qui explique la création des écoles Diwan en 1977, à la faveur d'un assouplissement de la législation organisant l'enseignement privé (destiné à l'origine à l'enseignement catholique essentiellement). Dans le même temps, la radicalité celticisante du courant 4 avait suscité dans la génération des baby-boomers un mouvement de rejet, survalorisant au contraire les parlers populaires truffés d'emprunts français. D'où une

42. Kevin RotTET, « Neology, competing authenticities and the lexicography of regional languages : The case of Breton », Dictionaries, t. 35, 2014. Je remercie Malo Morvan de m’avoir signalé cet article.

43. Roparz Hemon est à vrai dire assez difficilement " classable ». Fondateur et pilier de Gwalarn, il n'a pas de mots assez forts pour dire combien il est vital pour le breton de se départir de l'influence du français (cf. Roparz HEMON, Ar bed dre ar brę̧oneg, Lesneven, Hor Yezh, 2e édition, 1994 [1966], p. 138-139). De même, il salue avec enthousiasme la création du SADED, donne des comptes rendus élogieux des publications de Preder (ibidem p. 130,153-158) et use abondamment de leurs néologismes. Mais s'affranchir du français ne doit pas empêcher le discernement : il condamne les excès des puristes qui l'ont précédé, estimant qu'il ne faut pas renier les mots d'origine romane ou étrangère au seul motif de leur provenance (ibid. p. 166-167 ; Roparz HEMON, «Tri oadvezh ar brezhoneg ", Al Liamm, t. 26, 1951, p. 40-41) et propose des clés d'adaptation en breton pour les racines internationales (Roparz HEMON, " Gerioù etrevroadel », Hor Yez̧h, t. 12-16, 1957). Ce qui compte pour lui, c'est de faire du breton une langue moderne, apte à tout dire, sortie de sa sujétion au français, aux ressources internes revigorées. Mais pas expurgée de toute influence étrangère, fut-elle française. 
grande confusion sur la légitimité des termes à employer. Alors que les élèves du réseau Diwan approchent de l'entrée au collège, le flou des terminologies scientifiques, déjà handicapant en primaire, ne peut plus durer ${ }^{44}$. En 1985, des linguistes et enseignants du secondaire et du supérieur, notamment Lukian Kergoat, Francis Favereau et Yvon Gourmelon, se réunissent donc sous l'égide de l'association Kreizenn ar geriaouin afin de normer le breton scolaire. Globalement, ils entérinent certains néologismes déjà bien établis (issus du courant 3), mais recourent plus volontiers (surtout pour les créations nouvelles) à des termes internationaux bretonnisés : segmant, izoskelel, diametr, perimetr, paralelepiped, poligon, etc., qui se passent de traduction. Destinés au départ à Diwan, ces travaux donneront lieu à des publications à partir de 1989, qui auront un effet d'entraînement sur les autres enseignants. L'association continue ses activités sur internet (cf. supra), où elle semble pousser toujours plus loin ses choix translinguistiques (je reviendrai sur ce terme). Ils s'éloignent en cela de Francis Favereau, dont le dictionnaire, également très éclectique, reflète davantage la langue populaire du centre-Bretagne et ne ferme pas la porte aux emprunts directs au français.

6) L'ultime sursaut des "Bleus de Bretagne » (1986-2012): parallèlement à la création de Diwan par les baby-boomers, la génération précédente des bretonnants lettrés, la dernière à avoir eu le breton pour langue première $^{45}$ et qui n'entend pas se laisser déposséder, produit à son tour un dictionnaire en 1986 sous la direction de P.-J. Hélias. L'équipe d'auteurs fait le choix résolu de refléter une langue populaire. De ce fait, le dictionnaire se rapproche du courant 1 : les termes techniques et abstraits y sont peu nombreux et souvent imprécis. Parallélépipède est ainsi traduit par une périphrase aussi imagée qu'approximative : eur pez c'bweh fas (une pièce à six faces). Cette politique éditoriale n'est pourtant pas constante, puisqu'on y relève tout de même des néologismes savants : lieskementadur pour multiplication (apparu en 1919 sous la plume de Vallée). On y lit aussi kensturieg (créé par Roparz Hemon en 1970, sans

44. Lukian KERGOAT, op. cit.

45. Il y aura encore des bretonnants de langue maternelle bien après eux, mais cela ne concernera plus que des individualités et non une génération entière. La fracture se situant grosso modo vers 1945. 
doute d'après peurgensturieg pour parallélogramme chez Vallée en 1937, luimême probablement issu de kenstur créé par Laîné en 1934), mais cette fois avec le sens de parallèle! Quant à opération, la traduction proposée est operasion (aux côtés de jedadenn, cf. supra). L'addition, quant à elle, est rendue par kont (compte), qui traduit aussi calcul, avec l'exemple goud a ra mad konta (il sait bien compter), pour il sait bien calculer. Reflet fidèle et rabelaisien du breton paysan, il atteint donc vite ses limites lorsque le sujet devient pointu, notamment scientifique. On peut en dire autant du dictionnaire publié par Jean Le Dû en 2012, description d'un parler local, ignorant les novations lettrées des dernières décennies. Bien que les auteurs soient favorables à l'enseignement du breton, leurs choix ne semblent pas compatibles avec un usage scolaire et relèvent plus d'une démarche muséographique.

7) Le breton internationalisé: toujours du breton?(1995-...): de nouveaux courants se dessinent à partir du milieu des années 1990, lorsqu'avec la création de TES s'intensifie le travail des commissions de terminologie. La tendance translinguistique se renforce alors, portée par Jean Marot, mathématicien, professeur à l'Université de Brest. Après avoir joué un rôle important chez Diwan (courant 5), il poursuit dans sa voie : rejet des néologismes à base celtique, proposition de quantité de termes internationaux à la graphie bretonnisée. Le résultat de ses réflexions sera publié en 2002 dans un dictionnaire des mathématiques, à l'introduction très argumentée, qui met en parallèle breton, français, anglais, allemand et russe ${ }^{46}$. Ce parti pris translinguistique radical conduit toutefois à se demander si l'on a toujours affaire à du breton. Marot avance que devant les néologismes celtiques kelc'bdro (orbite), liestal (polyèdre), pevarzal (tétraèdre), troellenn (spirale), un mathématicien ne reconnaît pas son monde. Cependant, un bretonnant pourrait à bon droit faire remarquer que devant integrant (intégrant), diferañsiel (différentiel), ekwivalant (équivalent), invers (inverse), il ne reconnaît pas davantage sa langue...

8) Le breton comme ressource pédagogique (1995-...) : symétriquement à la tendance translinguistique, d'autres voix défendent le recours à la néologie à base celtique. Mais cette fois pour des raisons très diffé-

46. Jean MAROT, op. cit. 
rentes des courants nationalistes des années 1930. C'est en particulier le cas de J.-D. Robin, alors conseiller pédagogique dans l'enseignement public. Partant de son expérience d'instituteur de terrain et développant une réflexion nourrie sur la bilingualité des élèves ${ }^{47}$, il soutient l'idée d'utiliser au mieux les ressources propres des deux langues, qui peuvent ainsi apporter des éclairages différents et complémentaires sur les concepts ${ }^{48}$. Il invite donc à réhabiliter les termes celtiques, non par rejet du français, mais au motif de leur pertinence pédagogique. Doté d'une expertise limitée en mathématiques et d'une personnalité entière, qui n'incitera pas ses contradicteurs au compromis, ses positions n'auront pas l'audience et le crédit qu'elles auraient pourtant mérités.

Cette mise en perspective permet de mieux comprendre la prolixité du breton, qui est moins le fruit d'une richesse interne que de profondes divisions au sein du mouvement culturel breton : en matière de création lexicale, pratiquement tous les lexicographes actifs avant la fin du $\mathrm{XX}^{\mathrm{e}}$ siècle furent des voix prêchant dans le désert. Bien peu des termes énumérés plus haut seront sortis de la confidentialité et auront connu un usage réel. Le breton ayant été exclu de l'école et bientôt des médias, ces productions ne furent lues, entendues, évaluées et adoptées que par un petit cénacle de passionnés. Mais elles n'atteignirent jamais une large diffusion que seule une scolarisation de masse en breton aurait permise. C'est donc l'absence d'usage sociétal, de «marché linguistique » faisant spontanément le tri entre des mots surabondants qui explique un tel degré de variation. C'est aussi la raison pour laquelle le champ lexical de la géométrie en particulier apparaît si prolifique. Pour une société humaine, savoir dénombrer est une nécessité qui s'impose vite à partir d'un certain niveau de développement et d'échanges : compter les jours, le bétail, les hommes... est devenu une nécessité en Europe il y a plusieurs millénaires ${ }^{49}$. D’où le développement précoce

47. Josiane Hamers \& Michel Blanc, Bilingualité et bilinguisme, Bruxelles, Mardaga, 1983.

48. Jean-Dominique RoBIN, « L'enseignement (des mathématiques) en breton est-il une gageure? ?, Klask, t. 7, 2004.

49. Dans certaines langues d'Amazonie, il n'y a pas de numération au-dessus de deux : le décompte se limite à un, deux, plusieurs. La numération n'est pas nécessaire pour des populations isolées, qui ne connaissent qu'une seule saison, où par conséquent le temps est une éternelle reconduction du présent, ce qui rend vain même l'action de compter les jours. 
de systèmes de numération complexes, partagés à l'intérieur de chaque famille linguistique, parfois même dans l'ensemble des langues indoeuropéennes ${ }^{50}$. Mais le besoin de nommer et de penser des formes géométriques ne revêt pas la même évidence : dans une société paysanne, la forme d'un champ peut être approximative, sa superficie s'évaluer en journées de travail ou en productivité, bien plus dépendante de la nature du sol que de son étendue. La conceptualisation d'une figure abstraite par ses seules propriétés est donc quelque chose dont on peut fort bien se passer pendant des siècles. Et ceci se pérennisera d'autant plus facilement lorsque les besoins éducatifs sont couverts à partir d'un certain seuil d'abstraction par le recours à une autre langue (en l'occurrence le latin, puis le français). Nul besoin alors de développer une terminologie précise et univoque pour désigner un impensé, qui reste une non-réalité. Pour l'immense majorité des locuteurs adultes du breton, le lexique mathématique sera donc longtemps inexistant avant le développement de la scolarisation, puis simplement constitué d'emprunts spontanés au français, plus ou moins adaptés phonétiquement.

Ce défaut de communauté d'usage aura donc rendu aisé pour chaque linguiste (aux compétences variables) de contester tel ou tel terme et de proposer un vocable autre, de sa propre conception. Bien que n'ayant pas davantage de chances de le voir adopté à grande échelle. La plupart de ces tentatives lexicales possédant toutefois leur cohérence, il est compréhensible qu'un éditeur puisse raisonnablement hésiter et renoncer à trancher entre deux doublets. Ainsi, chaque mot proposé a pu laisser une trace, même si c'est une trace forcément discrète. L'adoption en 1989 pour addition et soustraction, de sammadenn/sammadur et lamadenn/lamadur représente donc une sorte de retour au bon sens, renouant avec la langue populaire attestée par le Catholicon de 1464 dans un cas, avec le dictionnaire de Grégoire de Rostrenen de 1732 dans l'autre. Il n'est d'ailleurs peut-être pas anodin que cette terminologie ait été adoptée dans le cadre de travaux des instances de Diwan, c'est-à-dire de la première mise en œuvre concrète et d'une certaine envergure d'un enseignement des mathématiques en breton.

50. /tri/ (et ses dérivés) représente le nombre trois dans quasiment toutes les langues depuis la Bretagne jusqu'à l'Inde du Nord. 


\section{Faut-il privilégier un lexique, et lequel ?}

En examinant les choix lexicaux des différents courants examinés supra, plusieurs jeux d'oppositions superposés se dessinent : certains lexicographes apparaissent comme globalement non-interventionnistes (courants 1 et 6 ). Leur but reste essentiellement descriptif, ils se contentent d'enregistrer le breton tel qu'il est effectivement parlé autour d'eux. D'où l'emprunt au français, langue de très longue date pourvoyeuse de termes abstraits, dans la pratique observable chez la majorité des locuteurs. Ce qui n'exclut pas totalement un recours à la néologie ou même à l'annexion de termes issus de courants pourtant philosophiquement très éloignés (cf. supra). Mais toujours de façon très parcimonieuse. À l'inverse, la majorité des lexicographes peut être considérée comme interventionniste: diagnostiquant l'incomplétude du vocabulaire mathématique breton, ils entendent remédier à cette situation par la création de vocables nouveaux. Cette nécessité étant actée, les interventionnistes se divisent à nouveau en deux grandes tendances. Les uns (courants 7 et 5, ce dernier avec réserves) optent pour un lexique translinguistique : à base gréco-latine, arabe etc., d'extension sinon universelle, du moins internationale. Le tout bretonnisé par l'adjonction de suffixes (perspektivenn, TES 2005) ou par la graphie (oktogon). Les autres (courants 2, 3, 4 et 8) sont au contraire partisans de néologismes à base de racines celtiques. D'où pour octogone : eiz-koñek et eiz-kornek en 1847 ; eizhkorneg en 1989. Chose importante, le développement de ces courants n'est pas chronologiquement linéaire : si les courants 1, 2 et 3 se succèdent, les suivants coexistent, parfois dans une certaine hostilité.

Avant d'étudier les arguments des uns et des autres, il faut d'emblée en écarter un, qui sert souvent à disqualifier le point de vue opposé : le nationalisme linguistique. Pour certains partisans du lexique celtique, utiliser des termes issus du français (car le français est le sas par lequel transitent les termes dits "internationaux») serait une sorte d'aveu d'ignorance ou de renonciation à pouvoir tout dire en breton. $\mathrm{Y}$ avoir recours porterait en germe la destruction du breton, rendu méconnaissable par la densité des emprunts français. Ce serait entériner l'état de domination que subit le breton, langue d'une population qui perdrait ainsi tous les jours un peu plus les ressorts de la créativité de sa langue 
et ne pourrait la faire évoluer qu'en empruntant au dominant. Pour les partisans du lexique translinguistique, au contraire, c'est par l'abus de néologismes celtiques que le breton devient méconnaissable et de plus en plus coupé de la réalité de ses locuteurs. Toute attitude de rejet du français peut s'apparenter à une tentative à réécrire l'histoire : on invente un breton idéal, tel qu'il aurait dû être s'il n'avait jamais subi le contact asymétrique du français. Ce faisant, on rejette le présent et les locuteurs du breton tel qu'il est ${ }^{51}$. En fonction des convictions, les néologismes celtiques seront donc connotés soit comme nationalistes, séparatistes, voire fascisants, soit comme authentiquement bretons et seuls légitimes; tandis que les termes translinguistiques apparaissent soit comme la marque d'un laxisme et d'une renonciation au destin national de la Bretagne, soit comme modernes et ouverts. Or il faut bien avoir conscience que de telles affirmations relèvent bien sûr de choix purement idéologiques et qu'elles sont absolument dénuées de pertinence s'agissant d'évaluer l'acceptabilité strictement linguistique ou mathématique d'un terme. Malheureusement, ce sont souvent de tels arguments qui orientent les débats et qui polluent la réflexion. Le poids de ces représentations étant mis de côté autant que possible, seuls les arguments mathématiques et pédagogiques en faveur des uns et des autres seront donc maintenant examinés.

\section{Le lexique translinguistique}

Le premier avantage de ce type de lexique est sa transférabilité. La ressemblance entre termes mathématiques d'une langue à l'autre présente une certaine garantie que les élèves qui auront acquis un tel lexique ne seront pas dépaysés et s'adapteront facilement s'ils doivent poursuivre ultérieurement des études scientifiques en contexte francophone, mais également anglophone, germanophone, etc. Outre les poursuites d'études à long terme, il permet aussi de prévenir les difficultés d'élèves qui seraient amenés à déménager et donc à quitter la filière d'enseignement en breton pour intégrer une classe en français. L'utilisation d'un lexique translinguistique permet ainsi d'éviter un risque que présente le lexique à base celtique, celui d'enfermer les élèves dans un vocabulaire hermétique, qu'ils devraient désapprendre 
tôt ou tard pour se confronter au langage des mathématiciens, qui est largement international.

Autres avantages, celui de la précision et de l'universalité. Créer une terminologie mathématique suppose de la part des linguistes une capacité de conceptualisation mathématique très pointue. Et de pouvoir trouver dans la langue cible les ressources pour l'exprimer. En optant pour un lexique à base gréco-latine (pour l'essentiel), on se décharge de cette réflexion, pour se ranger sous l'autorité des seuls mathématiciens. Ainsi, instituer en breton parallelenn, c'est se plier à la définition euclidienne du parallélisme, qui construit le concept comme statique. C'est aussi être sûr qu'ainsi les élèves travailleront dans un cadre commun à toute la communauté scientifique. Alors que traduire parallèle par kenstur, c'est introduire une notion de mouvement qui n'existe pas dans la communauté des mathématiciens (le mot stur signifiant initialement gouvernail, par extension direction. Kenstur signifie donc plutôt « avançant en même direction », « inscrit sur la même trajectoire »). La néologie à base translinguistique apparait donc comme un garde-fou, assurant que les termes donnés pour équivalents entre langues diverses ne seront pas dévoyés et référeront toujours bien aux mêmes signifiés.

\section{Le lexique à base celtique}

Parmi les partisans du lexique celtique, il faut distinguer nettement entre deux types de motivations très différentes. Les courants anciens qui se sont développés à partir du début du XIX ${ }^{\mathrm{e}}$ siècle (courants 2 et 3) et le courant 4, qui est toujours actif, voient dans l'emprunt ou l'adaptation de racines exogènes une sorte de marque de faiblesse liée au statut dévalué de la langue ou une surcharge inutile ${ }^{52}$. Le courant 8 , au contraire, ne refuse pas l'emprunt par principe, mais par réalisme didactique : pour des élèves qui ne sont pas familiers du grec, le mot pentagone peut rester longtemps un terme abstrait. Pourquoi alors le doubler par pentagonenn, qui ne dit rien de plus des propriétés de l'objet? Est-ce dans l'intérêt des élèves d'apprendre un double lexique composé de mots quasi-identiques, décorés de suffixes pour les rendre un peu moins étrangers à la langue bretonne? Alors que le néologisme pemtueg

52. De même que l'abus d'anglicismes en français peut agacer quand il semble une marque de pédanterie, de paresse linguistique, de superficialité ou de snobisme. 
(composé de pemp $+t u+-e g$, soit $:$ cinq + côté + suffixe substantival) permet à l'élève une compréhension analytique. Une telle propriété est infiniment précieuse, car les didacticiens des mathématiques, comme les praticiens de terrain que sont les enseignants, savent bien que les mathématiques posent justement comme défi que la compréhension des concepts peut sembler acquise en apparence, alors que l'élève ne fait que manipuler des procédures (et des mots) vides de sens, retardant de plusieurs années l'apparition des difficultés.

L'élaboration d'un lexique mathématique, plus opérationnel à destination des enfants, peut donc apparaittre comme parfaitement justifiée, au risque de l'introduction de notions, de nuances qui n'existent pas dans les termes les plus répandus. Un mot comme kenstur, appris et utilisé aux côtés de parallèle, ne fausse pas le concept visé, il enrichit la compréhension du parallélisme. Car sa conceptualisation euclidienne, c'est-à-dire statique, n'est pas la seule possible : la langue navajo ne peut ainsi décrire les formes qu'en tant que résultat d'un mouvement ${ }^{53}$. Les savoirs mathématiques dont elle est porteuse constituent ainsi une géométrie dynamique ${ }^{54}$ qui offre un réservoir de possibilités insoupçonné des mathématiciens formés exclusivement à l'école de la Grèce. Manier tour à tour kenstur et parallèle procure donc un avantage cognitif qui est la raison d'être du bilinguisme scolaire. Si l'on juge que kenstur et pemtueg encombrent inutilement la mémoire des élèves et qu'il serait plus judicieux de limiter le stock lexical, alors pourquoi apprendre gwenn, du, ru\%, qui n'expriment rien de plus que blanc, noir et rouge?

Enfin, l'argument du caractère international du lexique mathématique doit être fortement nuancé. Lorsque l'on examine le dictionnaire de J. Marot ${ }^{55}$, qui a l'avantage de mettre cinq langues en regard, on constate bien la transversalité de beaucoup de termes : discriminant, entropie, graphique, intervalle, multilinéaire, entre autres, sont communs aux quatre grandes langues scientifiques, à l'orthographe ou à la dérivation près. On note aussi une extrême proximité entre français et anglais. Mais l'allemand et le russe prennent souvent le large, comme on le voit

53. Bill BARTON, The language of mathematics, telling mathematical tales, New York, Springer, 2008, p. 28-33.

54. Ibidem.

55. Jean MAROT, op. cit. 
dans le tableau suivant. De diamètre, inférence, résoudre, distribution, isocèle et pentagone, seul le premier se rencontre en russe ${ }^{56}$, tous les autres étant rendus par des termes indigènes. Réalité qui se vérifie également dans d'autres grandes langues scientifiques comme le chinois et le japonais :

\begin{tabular}{|c|c|c|c|c|c|}
\hline & $\begin{array}{l}\text { Breton } \\
\text { (J. Marot) }\end{array}$ & Allemand & Russe & Chinois & Japonais \\
\hline Diamètre & diametr & Durchmesser & diametr & zhijìng & chokkei \\
\hline Inférence & inferañs & Schlußfolgerung & vyvod & tuì lì & suiron \\
\hline Résoudre & resolviñ & lösen & rešat' & jié jué & kaiketsu suru \\
\hline Distribution & distribussion & Verteilung & raspredelenie & fèn bù & bunpai \\
\hline Isocèle & isoskel & gleichschenklig & ravnobedrennyj & dèng yāo & nitōhen \\
\hline Pentagone & pentagon & Fünfeck ${ }^{57}$ & pjatingolnik & wü biān xíng & gokakei \\
\hline
\end{tabular}

L'arabe et le persan montreraient sans doute d'aussi forts contrastes. Quid alors des solutions avancées par Marot : diametr, inferañs, resolvin, distribussion, isoskel et pentagon? Puisqu'il n'y a pas de lexique international faisant consensus sur ces termes, ces choix apparaissent plus comme du français bretonnisé. Et si le latin et le grec (voire le français) doivent rester la source principale du lexique mathématique breton, pourquoi propose-t-il gwrizienn pour racine, kenfeurelenn pour proportionnelle, steudet pour aligné, heñveliez̧ pour similitude, antermenet pour indéfini, diskweler pour indicateur? La cohérence des choix lexicologiques, revendiquée par l'auteur, n'appellerait-elle pas plutôt des créations du type * rasinenn, *proporsionel, *alignet, *similitudenn, *indefinit et $*$ indikator? De même on a peine à croire que kedasparž (pour équirépartition) puisse voisiner dans le même ouvrage avec ekwidistañs (pour équidistance).

\section{Pour quels élèves travaille-t-on ?}

Au-delà des avantages respectifs des différentes terminologies, ce qui semble déterminant dans les choix des uns et des autres, c'est la

56. Par ailleurs, beaucoup de termes n'apparaissent qu'en français et en anglais, mais manquent en allemand et en russe : ascendant, cuspidal, matroïde, médian, partition, pavé...

57. L'allemand dispose aussi de Pentagon, mais le mot est peu usité car connoté en référence au ministère américain de la défense. 
conceptualisation même de l'enseignement des langues et de la prise en compte du plurilinguisme des élèves.

Ainsi qu'il a déjà été souligné, il n'est pas anodin que les efforts actuels en terminologie bretonne soient nés dans le sillage des écoles Diwan. Le mouvement impulsé à partir des années 1980 devait répondre à un besoin concret : créer pour un cadre scolaire, tout en anticipant l'acceptabilité sociale des termes proposés. Pour une population au bilinguisme asymétrique, dont le vocabulaire abstrait et scolaire n'était composé que de mots français, la bretonnisation de termes venus du français (plutôt que l'invention de mots indigènes, compréhensibles par analyse, mais éventuellement opaques au premier abord) assurait une continuité avec la langue populaire, rendant mieux acceptable un projet de scolarisation en breton ${ }^{58}$.

Mais surtout, les écoles Diwan ont comme particularité d'être immersives ${ }^{59}$. Le breton y est la langue de communication ordinaire, en classe et à l'extérieur de la classe. C'est aussi la seule langue d'enseignement durant les premières années de scolarisation (jusqu'au CE1). Le français est donc présent dans leur cursus, mais elles semblent avoir pour idéal implicite un enseignement monolingue bretonnant. Les mathématiques en tout cas y sont enseignées par le seul vecteur du breton. Tout cela au milieu d'une société massivement francophone. Les écoles Diwan ressemblent alors à autant d'îlots bretonnants dans un océan de français. Ce qui se traduit certes par un niveau de maitrise de la langue supérieur (à court terme) aux classes paritaires, mais fait aussi peser le risque d'un enfermement intellectuel et relationnel. On comprend alors la nécessité d'établir des passerelles avec le monde

58. Il faut d'ailleurs noter que les défenseurs des termes translinguistiques sont généralement des bretonnants premiers, ou connaissant de l'intérieur la civilisation bretonnante aujourd'hui disparue, alors que les partisans des néologismes celtiques ont souvent été des bretonnants seconds, ayant appris le breton tardivement, l'ayant peu pratiqué en société et affichant souvent un certain dédain pour les locuteurs populaires, en qui ils ne voyaient qu'un amas d'ignorants.

59. Inspirées en cela d'un modèle que des militants bretons avaient vu fonctionner au pays basque. L'enseignement en langues autres que l'espagnol (catalan et basque) existait déjà de façon clandestine sous Franco, à une assez large échelle. La fin de la dictature permit d'officialiser une pratique qui avait gagné ainsi ses lettres de noblesse et emmagasiné un très fort dynamisme. D'où la légitimité peu contestée de formes très volontaristes d'enseignement en basque et en catalan. 
extérieur. La meilleure passerelle étant, sur le terrain des mathématiques, l'adoption d'un lexique transparent d'une langue à l'autre.

Cette notion de transparence interlangue paraît le cœur de l'argumentation des défenseurs du lexique translinguistique. Mais elle est largement surévaluée. Certes, avec des termes proches, il y a transparence, dans le sens de transferts interlinguistiques aisés : qui connait les termes dans une langue, passera sans difficulté à l'autre. Mais ceci ne peut être vrai que pour un mathématicien déjà confirmé, qui maitrise bien les concepts auxquels les mots renvoient. Pour un mathématicien non-expert, comme le sont des élèves abordant une nouvelle notion, et a fortiori pour un élève en difficulté, un mot obscur dans une langue le restera dans l'autre. D'où la démarche inverse, la néologie, qui permet de s'appuyer sur une transparence intralinguistique. Là aussi, il est question de bâtir des passerelles, mais cette fois entre les mots et leur sens.

C'est là une démarche davantage défendue dans l'enseignement paritaire (public et privé catholique). Contrairement à Diwan, celui-ci a vocation à enseigner les disciplines dans les deux langues conjointement. Ce qui en permet une utilisation contrastive et complémentaire. Ce système reconnaît donc la bilingualité ${ }^{60}$ fondamentale des élèves : il vise à les équiper dans les deux langues de la manière la plus efficace. Si des élèves doivent poursuivre un cursus scientifique de haut niveau (ce qui ne sera pas le cas de tous), il n'y a guère de chance que ce soit en breton. C'est donc en ayant travaillé les mathématiques en français comme en breton, dès le plus jeune âge, qu'ils y seront les mieux préparés. De même pour les élèves devant intégrer une classe monolingue en français. L'enseignement bilingue n'est donc pas une sorte d'enseignement bas de gamme par rapport à Diwan, manquant d'audace ou d'ambition pour le breton. Pour Robin ${ }^{61}$, ses acteurs doivent prendre conscience de sa spécificité : au lieu de cloisonner les langues, il les fait dialoguer et mobilise chacune comme ressources pour la compréhension des concepts.

60. Josiane HAMERS \& Michel BlANC, op. cit.

61. Jean-Dominique RoBIN, op. cit. 


\section{Conclusions}

Quelles perspectives alors pour le lexique mathématique breton? La prolixité dont témoigne son passé, comme encore son présent, s'avère manifestement excessive. Au lieu d'attester la vitalité de la langue et sa capacité à exprimer les concepts les plus fins, l'imagination quasi sans borne de lexicographes travaillant en ordre dispersé, voire en opposition les uns aux autres, a plutôt abouti à une cacophonie. Situation d'autant plus dommageable qu'elle pourrait jeter un certain discrédit sur la possibilité d'une mise en œuvre efficace dans un cadre scolaire et par oblitérer davantage un avenir apparaissant déjà incertain du fait de la rétractation de l'usage sociétal de la langue. C'est donc dire si la nécessité est impérieuse d'une plus grande stabilité et d'un meilleur consensus. En adoptant un point de vue optimiste, il est toutefois possible d'avancer que consensus et stabilité sont désormais en bonne voie, grâce à la prééminence des choix lexicologiques opérés dans le cadre de TES, principal diffuseur de matériel mathématique en breton, et grâce au caractère collégial de ces décisions. Dans une large mesure, la cacophonie appartient donc au passé.

Mais il en restera probablement toujours quelque chose, puisqu'il n'existe pas d'instances normatives dotées d'une autorité indiscutable sur le breton. Même au sein de TES, le débat peut exister, notamment sur la priorité à donner à l'emploi de termes de construction celtique ou translinguistique. Il est donc important que la recherche contribue à éclairer ce type de débats. L'une de ses contributions pourrait d'ailleurs être, qu'en définitive, il n'est peut-être pas nécessaire de trancher entre les deux grandes options. Ainsi qu'il a déjà été brièvement mentionné plus haut, rappelons que l'allemand dispose d'un double lexique pour désigner les figures géométriques, l'un à base grecque : Pentagon, Hexagon, Oktagon; l'autre d'origine germanique: Fünfeck, Sechseck, Achteck. Que des lexicographes bretons considèrent un tel dualisme comme impossible ou non souhaitable, jetant l'anathème sur les terminologies qui n'ont pas leur faveur, est probablement le résultat le plus tangible de leur formation française. Et du fétichisme hérité d'une idéologie linguistique d'essence malherbienne: à chaque mot doit correspondre une chose et à chaque chose un mot. Les registres scientifique et mathématique en particulier seraient le lieu où un tel 
principe s'appliquerait le plus radicalement. Or c'est faire peu de cas de la réalité des langues, qui hors de France, peuvent offrir bien peu de prise à l'académisme, tout en permettant une pensée scientifique pointue, et peut-être d'autant plus inventive. L'anglais, première langue scientifique au monde, est ainsi parfois incroyablement imprécis : qui sait parmi les dogmatiques bretonnants qu'il n'y a pas de mot anglais pour dire dizaine, centaine et retenue ${ }^{62}$ ? De même, le gallois se montre bien peu embarrassé, lorsqu'il traduit losange par diemwnt, de l'anglais diamond (soit le diamant, qui est aussi outre-Manche le nom du carreau au jeu de cartes).

Un tel exemple rappelle que s'il existe un lexique plus ou moins international en mathématiques, ce n'est pas tant en vertu de l'universalité des concepts mathématiques, qu'en raison des accidents de l'histoire des langues et des relations que leurs locuteurs ont entretenues. Ainsi, le français ayant été la langue du pouvoir en Angleterre pendant plusieurs siècles, il en a résulté pour l'anglais non seulement une masse d'emprunts directs au français de termes abstraits, mais également la naturalisation des procédés français de dérivation du latin. D'où l'impression de miroir entre les deux langues, jusque dans des créations lexicales récentes. L'allemand se présente comme un peu plus original. D'une part parce que la flexion y est très productive, autorisant nombre de néologismes indigènes; et d'autre part parce que ses registres savants entretiennent un rapport plus pur à la latinité. Mais un simple facteur explique un certain niveau de perméabilité au vocabulaire français et anglais : la position géographique des pays de langue allemande face au mouvement de circulation des idées. Aux $\mathrm{XVI}^{\mathrm{e}}-\mathrm{XVII} \mathrm{e}^{\mathrm{e}}$ siècles, le dynamisme scientifique européen suit un axe allant de l'Italie à l'Angleterre; à partir du XVIII', il va de la Manche à la Baltique. Au moment où les pays du Nord, qui n'avaient raisonné auparavant qu'en latin, deviennent des centres d'activité scientifique, le français et l'anglais sont donc les seules langues européennes à être déjà les vecteurs d'une tradition scientifique mature (le néerlandais aurait pu être dans une position analogue, mais ses élites ont préféré

62. Les dictionnaires usuels que j'ai consultés donnent tens et hundred. Pour retenue, le Collins \& Robert 1995 ne parvient à donner le sens mathématique que grâce à l'exemple : N'oublie pas la retenue / Don't forget what to carry over. 
adopter comme langues intellectuelles les langues de large diffusion de leurs voisins, surtout le français ; l'italien est insensiblement relégué à la musique). C'est donc à ces traditions scientifiques qu'elles vont s'alimenter, empruntant d'autant plus facilement que le nationalisme, fut-il linguistique, n'existe pas encore, ou si peu. Après la Prusse, le Danemark, la Suède, viendra la Russie. Autant de pays, autant de langues, qui partagent un fonds lexical mathématique commun, mais qui s'étiole à mesure que l'on s'éloigne de la matrice franco-anglaise. En revanche, ce fonds lexical commun ne se rencontre quasiment pas dans des langues comme le tchèque, le polonais, le finnois et le hongrois (cf. annexe 4) : parlées à l'époque moderne dans des empires aux élites allophones, ces langues sont peu présentes, voire totalement absentes dans l'enseignement scientifique, où dominent l'allemand, le russe, le suédois, le français. C'est donc au XIX ${ }^{\mathrm{e}}$ siècle, lorsque ces langues font l'objet d'une promotion après qu'elles soient devenues le support d'une identité nationale, qu'elles sont dotées des terminologies qui leur faisaient défaut. En puisant dans le fonds indigène. Il s'agit là d'une sorte de réflexe que toutes les langues nouvellement promues semblent expérimenter, et qui correspond aux courants 2 à 4 identifiés pour le breton. Incriminer un prisme nationaliste de type xénophobe n'est pas forcément pertinent : en Nouvelle-Zélande, le maori a connu exactement la même évolution depuis les années $1980^{63}$, sans que l'on puisse la relier à un nationalisme maori d'exclusion. Ce qui parait primer serait plutôt la simple recherche de correction linguistique, ressentie comme menacée par l'omniprésence d'une autre langue, et non nécessairement la volonté de distanciation per se d'avec celle-ci. Les résultats obtenus peuvent être jugés maladroits, ils le sont seulement tant qu'un usage sociétal ne les a pas consacrés.

Reste la question de l'adéquation du vocabulaire à la chose nommée. Là encore, la recherche de perfection et le dogmatisme dont font preuve nombre de lexicographes bretonnants interrogent : certains semblent tenir que pour être acceptable, une terminologie bien construite doit être sémantiquement irréprochable. Écho d'un académisme typiquement français, alors que la resémantisation est un phénomène courant, y compris en mathématiques : bypoténuse signifie étymo-

63. Bill BARTON, op. cit., p. 1. 
logiquement [côté] placé en dessous. Le rapport entre signifiant et signifié est donc souple et la compréhension d'un concept n'est pas liée à une forme linguistique donnée. Du moins pour le mathématicien expert, ou l'élève qui a bien compris la leçon. Car pour un élève de primaire et particulièrement un élève en difficulté, il semble qu'un lexique déductible analytiquement représente une clé précieuse d'accès au sens. D’où encore une fois la comparaison avec l'allemand, qui parait un exemple intéressant de bonne pratique. Le double lexique y est en effet mis en œuvre en fonction d'impératifs didactiques : en primaire, le lexique germanique (Sechseck, Achteck...) sert à découvrir et manipuler les polygones, qui seront ensuite renommés selon la terminologie grecque (Hexagon, Oktagon...) en secondaire et dans l'enseignement supérieur. Est-il si extravagant de penser que le double lexique du breton pourrait être mobilisé avec fruit de la même façon? 


\section{ANNEXE 1. Relevé des termes présents dans les différents dictionnaires}

\begin{tabular}{|c|c|c|c|}
\hline Français & TES & Sources en ligne & Unanimité \\
\hline Nombre & niver & $\mathrm{O}, \mathrm{K}, \mathrm{P} \& \mathrm{~F}$ : niver & $x$ \\
\hline $\begin{array}{l}\text { Numéra- } \\
\text { tion }\end{array}$ & niveradur, niveroniezh & $\begin{array}{l}\mathrm{K} \& \mathrm{P}: \text { niveradur } \\
\mathrm{F}: \text { nivererezh }\end{array}$ & \\
\hline Calcul & $\begin{array}{l}1998 \text { : jedadenn, jedoniezh } \\
2005 \text { : jedad, jedadenn }\end{array}$ & $\begin{array}{l}\mathrm{O}: \text { jedad } \\
\mathrm{K}: \text { jedadenn, jederezh, jedoniezh } \\
\mathrm{P}: \text { jedadur, jediñ (verbe) } \\
\mathrm{F}: \text { jed, jederezh, jedadenn }\end{array}$ & \\
\hline Opération & oberiadenn, oberiadur & $\begin{array}{l}\mathrm{K} \text { : jedadenn, oberadenn } \\
\mathrm{P}: \text { jedadur, gwezhiadur jedoniel, gwezhiadenn } \\
\mathrm{F}: \text { operasion, jedadenn, oberiadur (abstr.) }\end{array}$ & \\
\hline Addition & $\begin{array}{l}1998 \text { : sammadur \& } \\
\text { sammadenn } \\
2005 \text { : sammadur, sammadenn, } \\
\text { sommadenn }\end{array}$ & $\mathrm{O}, \mathrm{K}, \mathrm{P} \& \mathrm{~F}$ : sammadur, sammadenn & \\
\hline $\begin{array}{l}\text { Soustrac- } \\
\text { tion }\end{array}$ & lamadur, lamadenn & O, K, P \& F : lamadur, lamadenn & $X$ \\
\hline $\begin{array}{l}\text { Multipli- } \\
\text { cation }\end{array}$ & $\begin{array}{l}1998 \text { : liesadur, liesadenn, } \\
\text { lieskementadenn, } \\
\text { lieskementadur } \\
2005 \text { : lieskementadenn, } \\
\text { lieskementadur }\end{array}$ & O, K, P \& F : liesadur, liesadenn & \\
\hline Retenue & dalc'h & $\begin{array}{l}\text { O, K \& F : dalc'h } \\
P: \text { dalc'had }\end{array}$ & \\
\hline Somme & $\begin{array}{l}1998 \text { : sammad } \\
2005 \text { : sammad, somm }\end{array}$ & $\begin{array}{l}\mathrm{O} \& \mathrm{P}: \text { sammad } \\
\mathrm{K}: \text { somm } \\
\mathrm{F}: \text { somm, soumm }\end{array}$ & \\
\hline Unités & unanenn & $\mathrm{O}, \mathrm{K}, \mathrm{P} \& \mathrm{~F}$ : unanenn & $x$ \\
\hline Dizaine & degad & $\begin{array}{l}\mathrm{K}: \text { degad } \\
\mathrm{P}: \text { degad, deg } \\
\mathrm{F}: \text { deg }\end{array}$ & \\
\hline Centaine & kantad & $\mathrm{K}, \mathrm{P} \& \mathrm{~F}$ : kantad & \\
\hline Chiffres & sifr & $\begin{array}{l}\text { O, K \& P : sifr } \\
F: \text { sifr, chifr }\end{array}$ & $x$ \\
\hline Fraction & kevrenn & $\begin{array}{l}\mathrm{K}: \text { kevrenn } \\
\mathrm{P}: \text { rann } \\
\mathrm{F}: \text { rann, fraksion }\end{array}$ & \\
\hline Carré & karrezenn & O, K, P \& F : karrezenn & $x$ \\
\hline Cercle & Kelc'h & O, K, P \& F : kelc'h, kerlenn & \\
\hline $\begin{array}{l}\text { Rectangle } \\
\text { (n) }\end{array}$ & $\begin{array}{l}1998 \text { : skouergorneg } \\
2005: \text { skouergorneg, } \\
\text { skwergorneg }\end{array}$ & $\begin{array}{l}O \text { : skouergorn } \\
\mathrm{K}: \text { reizhkogn } \\
\mathrm{P}: \text { reizhkorn, reizhkorneg } \\
\mathrm{F}: \text { hirgarrez, rektankl }\end{array}$ & \\
\hline
\end{tabular}




\begin{tabular}{|c|c|c|}
\hline $\begin{array}{l}\text { Rectangle } \\
\text { (adj) }\end{array}$ & 1998 : skouergornek & $\begin{array}{l}\text { K : reizhkogn, skwer, skwergorn, skwergornek } \\
\text { P : serzh, reizhkornek } \\
\text { F : rektankl }\end{array}$ \\
\hline Octogone & $\begin{array}{l}1998 \text { : eizhkorneg, eizhkostezeg } \\
2005 \text { : eizhkostezeg }\end{array}$ & $\begin{array}{l}\text { K : oktogon } \\
P \text { : eizhkorn, eizhtueg } \\
\text { F : eizh kogn }\end{array}$ \\
\hline Losange & romb & $\begin{array}{l}O: \text { romb } \\
K: \text { lozañj, romb } \\
P \text { : lankell } \\
F: \text { lozañj }\end{array}$ \\
\hline $\begin{array}{l}\text { Parallélo- } \\
\text { gramme }\end{array}$ & $\begin{array}{l}1998: \text { kensturieg } \\
2005 \text { : parallelogram }\end{array}$ & $\begin{array}{l}K \& F: \text { parallelogram } \\
P: \text { kensturieg }\end{array}$ \\
\hline $\begin{array}{l}\text { Parallélé- } \\
\text { pipède }\end{array}$ & $\begin{array}{l}1998: \text { kensturdaleg } \\
2005: \text { kensturdaleg, kensturieg, } \\
\text { parallelepiped }\end{array}$ & $\begin{array}{l}\text { O \& P : kensturdaleg } \\
\mathrm{K}: \text { parallelepiped } \\
\mathrm{F}: \text { paralelepiped, « pezh c'hwec'h tu » }\end{array}$ \\
\hline Aire & gorread & $O, K \& P$ : gorread \\
\hline Périmètre & $\begin{array}{l}1998 \text { : trolinenn \& trohed } \\
2005 \text { : trohed }\end{array}$ & $\begin{array}{l}\mathrm{K}: \text { perimetr } \\
\mathrm{P}: \text { amregad, amregenn } \\
\mathrm{F}: \text { muzul-tro }\end{array}$ \\
\hline Diamètre & $\begin{array}{l}1998 \text { : treuzkiz \& treuzkizenn } \\
2005 \text { : diametr \& treuzkiz }\end{array}$ & $\begin{array}{l}\text { O \& P : treuzkiz } \\
\mathrm{K}: \text { diametr } \\
\mathrm{F}: \text { treuz, diametr }\end{array}$ \\
\hline
\end{tabular}




\section{ANNEXE 2. Sources examinées systématiquement}

\begin{tabular}{|c|c|}
\hline 1464 & Catholicon, Jean Lagadeuc (édition Quillivere de 1521 sur Gallica.fr) \\
\hline 1626 & $\begin{array}{l}\text { Colloques françois et breton traduits de françois en breton par G. Quiquer de } \\
\text { Roscoff, Morlaix, Georges Allienne. }\end{array}$ \\
\hline 1659 & Le Sacré Collège de Jésus, Julien Maunoir, Quimper, Jean Hardouin \\
\hline 1723 & $\begin{array}{l}\text { Dictionnaire breton-françois du diocèse de Vannes, Pierre de Châlons, } \\
\text { Vannes, Jacques de Heuqueville. }\end{array}$ \\
\hline 1732 & $\begin{array}{l}\text { Dictionnaire français-celtique ou françois-breton, Grégoire de Rostrenen, } \\
\text { Rennes, Vatar. }\end{array}$ \\
\hline 1744 & $\begin{array}{l}\text { Dictionnaire françois-celtique ou françois-breton du diocèse de Vannes, Claude- } \\
\text { Vincent Cillart de Kerampoul, Leyde. }\end{array}$ \\
\hline 1821 & $\begin{array}{l}\text { Dictionnaire celto-breton ou breton-français, Jean-François Le Gonidec, } \\
\text { Angoulême, Trémeau. }\end{array}$ \\
\hline 1842 & Dictionnaire français et celto-breton, Amable Troude, Brest, Lefournier. \\
\hline 1847 & $\begin{array}{l}\text { Dictionnaire français-breton de Le Gonidec enrichi par Th. Hersart de la } \\
\text { Villemarqué, Saint-Brieuc, Prud'homme. }\end{array}$ \\
\hline 1850 & $\begin{array}{l}\text { Dictionnaire breton-français de Le Gonidec enrichi par Th. Hersart de la } \\
\text { Villemarqué, Saint-Brieuc, Prud'homme. }\end{array}$ \\
\hline 1862 & $\begin{array}{l}\text { Manuel breton-français, classé par ordre de matières à l'usage des écoles } \\
\text { primaires, Joseph-Marie Toullec, Chavignaud. }\end{array}$ \\
\hline 1886 & Dictionnaire français-breton, Henri du Rusquec, Morlaix, Chevalier. \\
\hline 1904 & $\begin{array}{l}\text { Dictionnaire breton-français du dialecte de Vannes, Emile Ernault, Vannes, } \\
\text { Lafolye. }\end{array}$ \\
\hline 1919 & $\begin{array}{l}\text { Vocabulaire français-breton de Jean-François Le Gonidec. Nouvelle édition } \\
\text { mise à jour et considérablement augmentée par François Vallée, Saint-Brieuc, } \\
\text { Prud'homme. }\end{array}$ \\
\hline 1927 & $\begin{array}{l}\text { Gériadurig brezonek-gallek, vocabulaire breton-français, Emile Ernault, } \\
\text { Saint-Brieuc, Prud'homme. }\end{array}$ \\
\hline 1928 & Geriadurig-dourn brezonek-gallek, Roparz Hemon, Brest, Gwalarn. \\
\hline 1931 & $\begin{array}{l}\text { Grand dictionnaire français-breton, François Vallée, Rennes, Imprimerie } \\
\text { commerciale de Bretagne. }\end{array}$ \\
\hline 1934 & Mentoniez, Kerjean (Célestin Laîné), Brest, Gwalarn. \\
\hline 1937 & $\begin{array}{l}\text { Mots français et bretons classés d'après le sens, François Vallée, Carhaix, } \\
\text { Armorica. }\end{array}$ \\
\hline 1941 & Dictionnaire de poche français-breton, Roparz Hemon, Brest, Gwalarn. \\
\hline
\end{tabular}




\begin{tabular}{|c|c|}
\hline 1948 & Geriadur brezhoneg-galleg, Roparz Hemon, Al Liamm (2e édition). \\
\hline 1964 & Geriadur brez̧̧oneg-galleg, Roparz Hemon, Al Liamm (3e édition). \\
\hline 1970 & Geriadur brez̧honeg-galleg, Roparz Hemon, Al Liamm (4édition). \\
\hline 1970 & Dictionnaire français-breton, Roparz Hemon, $\mathrm{Al}$ Liamm (3e édition). \\
\hline 1985 & Geriadur brezhoneg-galleg, Roparz Hemon, Al Liamm (7e édition). \\
\hline 1986 & $\begin{array}{l}\text { Dictionnaire breton : breton-français / français-breton, Visant Seité, Andreo } \\
\text { ar Merser, Armand Keravel, Per Jakez Helias, Paris, Garnier. }\end{array}$ \\
\hline 1989 & $\begin{array}{l}\text { Geriaoneg galleg/brezhoneg, brezhoneg/galleg : matematik, Lukian Kergoat } \\
\text { (dir.), Eil derez Diwan. }\end{array}$ \\
\hline 1992 & $\begin{array}{l}\text { Geriaoneg galleg/brezhoneg, brezhoneg/galleg: matematik, Lukian Kergoat } \\
\text { (dir.), Eil derez Diwan (2e édition). }\end{array}$ \\
\hline 1993 & Geriadur bręhoneg-galleg, Roparz Hemon, Al Liamm (8e édition). \\
\hline 2002 & $\begin{array}{l}\text { Geriadur ar matematikoù - dictionnaire multilingue des mathématiques, Jean } \\
\text { Marot, Morlaix, Skol Vreizh. }\end{array}$ \\
\hline 2005 & Geriadur brezhoneg-galleg, Roparz Hemon, Al Liamm (11e édition). \\
\hline
\end{tabular}

\section{Autres sources consultées :}

\begin{tabular}{|c|l|}
\hline 1450 & $\begin{array}{l}\text { An Dialog etre Arzur Roe d'an Bretouned ha Guynglaff, Hor Yezh n } 200 \\
\text { \& 212. }\end{array}$ \\
\hline 1752 & $\begin{array}{l}\text { Dictionnaire de la langue bretonne, Dom Louis Le Pelletier, Paris, } \\
\text { Delaguette. }\end{array}$ \\
\hline 1915 & $\begin{array}{l}\text { Deviza ar er Régl hag ar en Induljanseu a drivet urh Sant Fransez, T. Ivon, } \\
\text { Lorient, Le Bayon. }\end{array}$ \\
\hline 1993 & $\begin{array}{l}\text { Geriadur bihan brezhoneg-galleg, galleg-brezhoneg. Dictionnaire élémentaire } \\
\text { breton-français, français-breton, Per Denez, Lesneven, Mouladurioù hor } \\
\text { yezh. }\end{array}$ \\
\hline 2012 & $\begin{array}{l}\text { Jedoniez̧, Yann-Baol an Noalleg, Preder. } \\
\text { palantionnaire français-breton, Martial Ménard, Quimper, Éditions }\end{array}$ \\
\hline 2012 & $\begin{array}{l}\text { Le trégorrois à Plougrescant, dictionnaire breton-français / français-breton, } \\
\text { Jean Le Dû, Brest, Emgleo Breiz. }\end{array}$ \\
\hline
\end{tabular}


ANNEXE 3. Extrait des tableaux de comparaison entre dictionnaires

\begin{tabular}{|c|c|c|c|}
\hline $\begin{array}{c}\text { TES } \\
\text { (1998 et } 2005)\end{array}$ & $\begin{array}{l}\text { 1re attestation } \\
\text { (sens } \\
\text { quelconque) }\end{array}$ & $\begin{array}{l}\text { 1re attestation } \\
\text { (sens identique } \\
\text { à TES) }\end{array}$ & $\begin{array}{c}\text { Divers } \\
\text { (variantes, étymologie, orthographe } \\
\text { d'origine...) }\end{array}$ \\
\hline $\begin{array}{l}\text { Niver } \\
\text { (nombre) }\end{array}$ & 1464 & 1464 & $\begin{array}{l}\text { Etym < latin } \\
1931 \text { : nombre entier : niver anterin ; } \\
\text { nombreuses autres entrées (nombre } \\
\quad \text { fractionnaire, négatif etc.) }\end{array}$ \\
\hline $\begin{array}{l}\text { Niveradur \& } \\
\text { niveroniezh } \\
\text { (numération) }\end{array}$ & $\begin{array}{c}1732 \\
\text { (niveradur) } \\
\\
1928 \\
\text { (niveroniezh) }\end{array}$ & $\begin{array}{c}1937 \\
\text { (niveradur) } \\
\\
1995 \\
\text { (niveroniezh) } \\
\text { (manuel Penaos } \\
\text { 'mañ kont?) }\end{array}$ & $\begin{array}{l}1732: \text { niveradur = dénombrement } \\
1821: \text { niveridigez = dénombrement } ; \text { nivererez = } \\
\text { dénombrement, énumération \& calcul } \\
1847: \text { nivererez \& niveridigez = dénombrement } \\
1850: \text { nivererez = action de dénombrer } \\
1927: \text { niverouriez } \\
1928: \text { nivererez (niveradur = dénombrement ; } \\
\text { niveridigez = recensement ; niveroniez \& } \\
\quad \text { niverouriez = arithmétique) } \\
1931: \text { nivererez \& niverouriez } \\
1937: \text { niveradurez \& niveradur (niveroniez = } \\
\quad \text { arithmétique) } \\
1986: \text { niveradur } \\
1989: \text { niveradur }\end{array}$ \\
\hline $\begin{array}{l}\text { Degad } \\
\text { (dizaine) }\end{array}$ & $1450(\mathrm{dec})$ & 1450 (dec) & $\begin{array}{l}1450: \text { dec (v. } 68) \\
1723: \text { un déc scoüét = une dizaine d'écus } \\
1732: \text { dizaine = un decq ( } \& \text { un decq-bennac) } \\
1904: \text { dégad } \\
1915 \text { (p.146) : vingtaine, cinquantaine, } \\
\text { soixantaine = uigentat, hanterhantat, } \\
\text { tri-uigentat } \\
1919: \text { dizaine = deg, pl. degou ; par dizaines : } \\
\text { a-zegou } \\
1927: \text { degad > signalé comme vannetais } \\
1931: \text { degad > régulier (langue commune) }\end{array}$ \\
\hline $\begin{array}{l}1998: \text { trolinenn } \\
\& \text { trohed } \\
2005: \text { trohed } \\
\text { (périmètre) }\end{array}$ & $\begin{array}{l}1931 \text { (trolinenn) } \\
1989 \text { (trohed) }\end{array}$ & $\begin{array}{c}1931 \text { (trolinenn) } \\
1989 \text { (trohed) }\end{array}$ & $\begin{array}{l}1732 \text { : tro } \\
1821 \text { : tro (circonférence) } \\
1927 \text { : reg = rayon tracé en terre par la charrue, } \\
\quad \text { de <rec>, } 1744: \text { : }\{\text { ligne de\} partage } \\
\quad \text { pour semer les différentes espèces » } \\
\quad \text { (article « sol ») ; également « sole ». } \\
1931 \text { : tro, trolinenn } \\
1934 \text { : trôad } \\
1970 \text { f-b : tro-linenn \& hed-tro } \\
1989: \text { trohed } \\
1998 \text { : amregad }\end{array}$ \\
\hline
\end{tabular}




\begin{tabular}{|c|c|c|c|}
\hline $\begin{array}{l}1998: \text { treuzkiz } \\
\& \text { treuzkizenn } \\
2005: \text { diametr } \\
\& \text { treuzkiz } \\
\text { (diamètre) }\end{array}$ & $\begin{array}{l}1919 \text { (treuzkiz) } \\
1989 \text { (diametr) }\end{array}$ & $\begin{array}{l}1919 \text { (treuzkiz) } \\
1989 \text { (diametr) }\end{array}$ & $\begin{array}{l}1732 \text { : définition (pas de terme) } \\
1842 \text { : définition et périphrase } \\
1847:<\text { Gall. trévuzul } \\
1862 \text { (p.152) : drevuzul \& digorder (avec } \\
\text { français entre parenth.) } \\
1886 \text { : linen-treuz \& rouden-treuz } \\
1919: \text { treuz, treuzlinenn, treuzkizenn } \\
1927: \text { treuskiz = communication, } \\
\text { rigole > treuskiziat (verbe) et mont } \\
\quad \text { a-dreuskiz = passer en travers ; } \\
\quad \text { treuskizenn = diamètre } \\
1934: \text { kreizer } \\
1986 \text { : treuz \& linenn-dreuz } \\
1989: \text { treuzkiz \& diametr }\end{array}$ \\
\hline
\end{tabular}

ANNEXE 4. Comparaison de quelques termes mathématiques en d'autres langues

\begin{tabular}{|l|l|l|l|l|l|}
\cline { 2 - 5 } \multicolumn{1}{l|}{} & Suédois & Finnois & Tchèque & Polonais & Hongrois \\
\hline Diamètre & diameter & halkaisija & průměr & średnica & átmérö \\
\hline Périmètre & omkretsen & kehä & obvod & obwód & kerülete \\
\hline Octogone & oktagon & kahdeksankulmio & osmiúhelník & ośmiokąt & nyolcszög \\
\hline Losange & diamant & timantti & diamant & diament & gyémánt \\
\hline Parallèle & parallell & rinnakkainen & paralelní & równoległy & párhuzamos \\
\hline Parallélépipède & parallellepiped & suuntaissärmiö & rovnoběžnostě & równoległościan & paralelepipedon \\
\hline
\end{tabular}

